\title{
The interaction effects between droplets condensing from moist air using a distributed point sink method
}

\author{
Shaofei Zheng ${ }^{1, *}$, Ferdinand Eimann ${ }^{1}$, Christian Philipp ${ }^{1}$, Tobias Fieback ${ }^{1}$, and Ulrich Gross $^{1}$ \\ ${ }^{1}$ Insititute of Thermal Engineering, TU Bergakademie Freiberg, 09599 Freiberg, Germany
}

\begin{abstract}
In modelling dropwise condensation, growth of the droplet is frequently assumed in an isolated form. However, for dropwise condensation of moist air the vapor diffusion from the surrounding to the droplet surface will be tremendously influenced by the blocking effect of the neighboring droplets. The influenced spatial distribution of water vapor totally determines a different condensation rate comparing with that by the isolated droplet model. In this work, a distributed point method (DPSM) as the method of Green function is developed to capture the interaction effects of droplets without requiring solution of the diffusion equation and the numerical discretization. Due to its nature, the automatically satisfied boundary conditions make sure the solution accuracy based on the uniqueness theorem. The significant characteristics for the interaction effects between droplets are investigated by handling a series of droplet arrays. During dropwise condensation, a typical droplet array containing up to 1000 droplets is considered. The results indicate that the interaction effect between droplets is critical in accurately predicting the condensation behavior for dropwise condensation in the presence of non-condensable gas.
\end{abstract}

\section{Introduction}

In dropwise condensation community, one important and challenging aspect is the presence of non-condensable gas (NCG) which apparently reduces its heat transfer coefficient [1-2]. In contrast to the case of pure steam, the vapor molecules must diffuse toward the vapor/liquid interface and then condense on the droplet surface, which is critical during condensation. Consequently, mechanism understanding for the simultaneous heat and mass transfer through the droplet from the surrounding to the condensation substrate becomes to be a notable aspect during dropwise condensation with considering NCG [1].

During dropwise condensation, the spatio-temporaldependent droplet growth correspondingly brings a complicated spatio-temporal dynamics behavior of droplets [3]. As because of its complexity, in studying dropwise condensation, droplet systems are generally described as isolated growing droplet in history [3-4]. This reasonable simplification has urged a great progress in dropwise condensation community. With this idea in mind, in our previous work [5-6], a multi-scale growth model has been proposed for dropwise condensation in the presence of NCG.

For heat and mass transfer driven dropwise condensation in case of NCG, there is a specific aspect which cannot be captured exactly. That is the interaction effect between droplets. The blocking effects between droplets for the vapor diffusion can significantly reduce their condensation rate [7]. With the description of the isolated growth, the droplet condensation rate is uniquely depended on its size. However, it will not be accepted due to the interaction effects. Ucar et al. [8] experimentally found that the condensation rate of one droplet surrounded by others in close proximity is 14$40 \%$ lower than that of a single isolated growing droplet. Similarly, in spite of the same size, a droplet far away from the large droplet grows $20 \%$ faster than a droplet neighboring large droplets, according to the experimental works by Leach et al. [9]. The explanation is the blocking of the vapor diffusion by each other. It is no doubt that the isolated droplet growth model will overpredict the condensation rate of droplets. Recently, using a semi-analytical point sink method, the interaction effects between droplets are reasonably captured and the predicted droplet condensation rate keeps consistent with the experimental data [10-11].

During dropwise condensation, a large number of droplets locating a widely size range are closely formed over the condensation substrate, which promotes quite strong interaction effects. Aiming to accurately define the strong interaction effects between droplets and the overall condensation rate over the whole substrate, a creative distributed point sink method (DPSM) is contributed in this work.

\section{The problem description}

In this subsection, the problem is described with some reasonable assumptions. For the droplets on a substrate, as shown in Fig. 1, vapor condensation is driven by the vapor concentration difference between the surrounding and their surfaces $c_{d s}-c_{\infty}$. The problem is simplified following $[10,12]:(1)$ the droplets are spherical and they have the same surface vapor concentration cds (namely the same surface temperature); (2) the condensation

\footnotetext{
* Corresponding author: shaofei.zheng@tttd.tu-freiberg.de
} 
process is seen as a near quasi-steady state which means the vapor concentration field is rapidly adjusted in comparison with changes of the droplet surface. Introducing the dimensionless vapor concentration, $C$, defined as:

$$
C=\left(c-c_{d s}\right) /\left(c_{\infty}-c_{d s}\right)
$$

Under these conditions, the vapor diffusion is governed by the Laplace equation following [10, 12]:

$$
\nabla^{2} C(\boldsymbol{r})=0
$$

with the boundary conditions $C_{d s}=0$ on all droplets surfaces and $C_{\infty}=1$ at infinity.

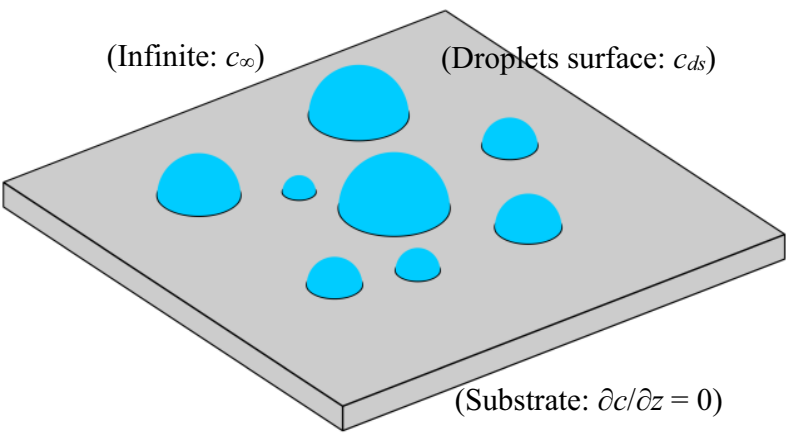

Fig. 1. Illustration of multiple condensation droplets over the substrate.

\section{Method development}

In order to quantitatively define the influence of the inter-droplet interaction on the condensation rate, the correction factor reflecting the interaction intensity is introduced by:

$$
\eta=m_{\text {sys }} / m_{\text {iso }}
$$

where $m_{s y s}$ and $m_{i s o}$ denote the droplet condensation rate within the droplet system and the isolated growth, respectively.

\subsection{Condensation of an isolated droplet}

Using the boundary conditions, the mathematics-based solution of the Laplace equation can give the droplet condensation rate based on the isolated-form growth [5, 12]:

$$
m_{i s o}=2 \pi(1-\cos \theta) R D\left(C_{\infty}-C_{d s}\right)
$$

where $\theta$ is the droplet contact angle, $\mathrm{R}$ means the spherical radius corresponding to the droplet and $D$ represents the vapor diffusion coefficient. For any contact angle, Eq. (4) provides the analytical solution for the condensation rate of a single droplet.

\subsection{Condensation of an isolated droplet}

For the field problem, a representing solution method is the method of Green's function, which is based on the uniqueness theorem that certain boundary conditions mathematically determine a unique solution. In general, a special "field" and the "source" producing it can be physically corresponded to a mathematic-physical equation, such as the temperature field and the heat flow rate ("source") by the equation of heat conduction. Consequently, the "field" can be defined by different arrangements of the "source" as long as certain boundary conditions are satisfied. Historically, the point sink/source method $[10-11,13]$ and the method of image [14] in handling the condensation/evaporation of droplets are all within the category of the method of Green's function.

In solving the field problem including the ultrasonic, electrostatic and magnetic field, the distributed point source method as a method of Green's function has been successfully developed as a powerful and invaluable analytical tool [15]. Without requiring numerical discretization of the interesting region and the boundary, the "source" faces/interfaces are replaced by a distribution of point sources. Strengths of the point sources are adjusted through the matrix formulation in a way that the boundary conditions can be accurately satisfied.

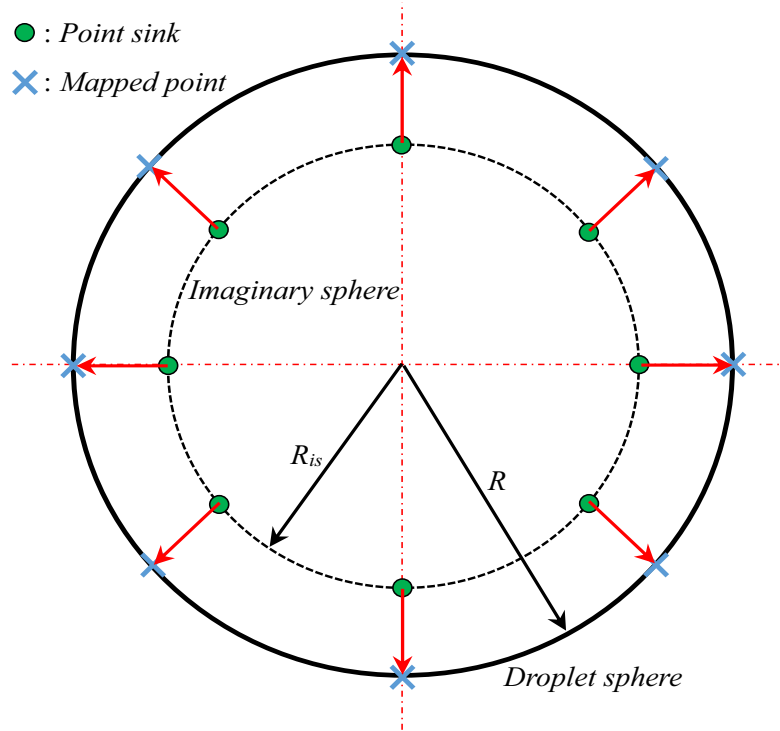

Fig. 2. Illustration of the distribution of the point sinks and the target points.

Correspondingly, herein the distributed point sink method (DPSM) is developed for solving the condensation behavior within a multiple condensing droplets system. The basic principle for the DPSM method is illustrated in Fig. 2. For each condensing droplet, its surface can be treated as a "source" surface which is responsible for the vapor concentration field outside the droplets. Aiming to replace the "source" surface, the point sinks are averagely distributed on an imaginary spherical surface which shares the same center with the droplet sphere. The positions of the point sinks are decided by the radius of the imaginary spherical surface $R_{i s}$. Considering $\mathrm{N}$ droplets and $M$ point sinks for each droplet, $M^{*} N$ point sinks are generated. For each point sink having the location vector $\boldsymbol{r}_{i}$, the Laplace equation becomes the Poisson equation by including the point sink term:

$$
\nabla^{2} C(\boldsymbol{r})=\sigma_{i} \delta\left(\boldsymbol{r}-\boldsymbol{r}_{i}\right)
$$

where $\sigma_{i}$ represents the strength of the point sink and $\delta(\boldsymbol{r}$ - 
$\boldsymbol{r}_{i}$ ) is the Dirac-delta function. At an arbitrary point $\boldsymbol{r}$, the normalized vapor concentration produced by this point sink $C_{i}(\boldsymbol{r})$ can be defined by integrating Eq. (5):

$$
\sigma_{i} /\left|\boldsymbol{r}-\boldsymbol{r}_{i}\right|=C_{\infty}-C_{i}(\boldsymbol{r})
$$

For the unknown strengths of $M^{*} N$ point sinks, one need to construct $M^{*} N$ equations. Hereby, the point sinks are mapped to the corresponding droplet surface forming $M^{*} N$ target points. Due to the linearity of the Laplace equation, the final concentration on each target point can be obtained by the superposition of the concentration induced by all point sinks:

$$
\sum_{i=1}^{N^{*} M} \sigma_{i} /\left|\boldsymbol{r}_{d s}-\boldsymbol{r}_{i}\right|=C_{\infty}-C\left(\boldsymbol{r}_{d s}\right)=1
$$

where $\boldsymbol{r}_{d s}$ is the location vector of the target point on the droplet surface. According to the boundary condition, the same normalized concentration on the droplet surface $C\left(\boldsymbol{r}_{d s}\right)=C_{d s}=0$, in Eq. (7) only the intensity of the point sink is unknown. Considering Eq. (7) for $M^{*} N$ target points, the following matrix equation can be constructed:

$$
\boldsymbol{A}\left(\begin{array}{c}
\sigma_{1,1} \\
\vdots \\
\sigma_{M, 1} \\
\vdots \\
\sigma_{M^{*}(N-1)+1, N} \\
\vdots \\
\sigma_{M^{*} N, N}
\end{array}\right)=\left(\begin{array}{c}
1-C_{d s}^{1,1} \\
\vdots \\
1-C_{d s}^{M, 1} \\
\vdots \\
1-C_{d s}^{M^{*}(N-1)+1, N} \\
\vdots \\
1-C_{d s}^{M^{*} N, N}
\end{array}\right)
$$

In Eq. (8), the right-side matrix is a unit column vector which denotes the boundary condition; the first term in the superscript indexes the target point on the surface of the droplet represented by the second term. For the leftside second term, the component $\sigma_{i, j}$ in the column vector is the intensity of the point sink $i$ on the imaginary spherical surface $j$. The coefficient matrix $\boldsymbol{A}$ is a square matrix having the order $N^{*} M$; its component $A^{j}{ }_{, i}$ is defined as the reciprocal of the distance between the point sink $\mathrm{i}$ and the target point $j$ :

$$
A_{i}^{j}=1 /\left|\boldsymbol{r}_{j}-\boldsymbol{r}_{i}\right|
$$

where $\boldsymbol{r}_{j}$ is the location vector of the target point $j$ and $\boldsymbol{r}_{i}$ is the position vector of the point sink $i$. The matrix $\boldsymbol{A}$ is known after choosing the radius of the imaginary sphere. With the known concentration of all target points, Eq. (8) is a linear system having $N^{*} M$ equations and $N^{*} M$ unknown intensities $\sigma$ of the point sinks. Finally, the problem becomes the solution of a linear system which provides the strengths of all point sinks.

Through the known positions and the solved strengths of all point sinks according to the boundary conditions, the vapor concentration in the interesting domain can be obtained by the superimposing method:

$$
\begin{gathered}
C(\boldsymbol{r})=1-\sum_{i=1}^{N^{*} M} f\left(\boldsymbol{r}, \boldsymbol{r}_{i}, \sigma_{i}\right) \\
f\left(\boldsymbol{r}, \boldsymbol{r}_{i}, \sigma_{i}\right)=\sigma_{i} /\left|\boldsymbol{r}-\boldsymbol{r}_{i}\right|
\end{gathered}
$$

where the function $f\left(\boldsymbol{r}, \boldsymbol{r}_{i}, \sigma_{i}\right)$ denotes the contribution of the point sink $\left(\boldsymbol{r}, \sigma_{i}\right)$ for the concentration at the point $\boldsymbol{r}$. Based on the Gauss's flux theorem, the condensation rate of droplet $p m_{s y s, p}$ within the droplet system can be defined by integrating the gradient of the concentration field over the droplet surface $S_{p}$ in the spherical coordinate system $(r, \xi, \varphi)$ :

$$
\begin{aligned}
m_{s y s, p}= & -\left.D \int_{S_{p}} \nabla C\right|_{R_{p}} d S_{p}=D \sum_{i=1}^{N^{*} M} f \mathcal{L}\left(R_{p}, \boldsymbol{r}_{i}, \sigma_{i}\right)(12 \\
& f_{\mathcal{C}}\left(R_{p}, \boldsymbol{r}_{i}, \sigma_{i}\right)= \\
& \int_{0}^{2 \pi} \int_{0}^{\theta}\left(\frac{\partial f(r, \xi, \varphi)}{\partial r}\right)_{r=R_{p}} R_{p}^{2} \sin \xi d \xi d \varphi
\end{aligned}
$$

with the function $f c\left(R_{p}, \quad \boldsymbol{r}_{i}, \sigma_{i}\right)$ representing the condensation rate of the droplet $p$ contributed by the point $\operatorname{sink}\left(\boldsymbol{r}_{i}, \sigma_{i}\right)$. And $f(r, \xi, \varphi)$ is the form of the function $f(\boldsymbol{r}, \boldsymbol{r}$, $\left.\sigma_{i}\right)$ in the spherical coordinate system.

When handling a large number of droplets, the DPSM method will result in a huge and dense linear system and a huge computing times of the surface integral. For example, considering $N=2000$ droplets and $M=200$ point sinks for each droplet, the dimension of the matrix equation in Eq. (8) and the time of the surface integral in Eq. (12) will be up to $N^{*} M=400,000$ and $N^{*} N^{*} M=8,000,000$, respectively. The solution suffers the problems of the computer memory and the computing time. Therefore, the generalized product-type biconjugate gradient method (GPBiCG) [16] as an iterative method is considered to solve the huge and dense linear system; and the TwoD algorithm based on the titled method [17] is utilized for the numerical evaluation of the surface integral. Finally, for improving the computation efficiency, a Fortran 90 code actualizing the GPBiCG and TwoD algorithms is paralleled using the open MPI.

To sum up, without requiring numerical solution of the diffusion-Laplace equation the DPSM method constructs a matrix formulation for predicting the condensation behaviors of multiple droplets. The model can capture the concentration distribution and the droplet condensation rate, as well as the interaction effects between droplets. Through defining the intensities of the point sinks by the boundary conditions, the given boundary conditions are satisfied exactly and automatically. Therefore, according to the uniqueness theorem, the solution accuracy can be guaranteed in DPSM.

\section{Results and discussion}

\subsection{Condensation of an isolated droplet}

It is no doubt that the DPSM method is also suitable for a single condensation droplet. As given in Eq. (4), the mathematics-based solution of the Laplace equation provides the analytical solution for the condensation rate of an isolated growth droplet. For validation, this section compares the predicted condensation rates for a single 
condensation droplet in different contact angles by the DPSM method against the analytical solutions. The case considers a single droplet on the substrate with the radius $R$ $=300 \mu \mathrm{m}$. The humid air at the temperature $T_{\text {air }}=303.15 \mathrm{~K}$ has a relative humidity $94 \%$. The temperature of the droplet surface is assumed to be equal to the substrate temperature $T_{d s}=300.1 \mathrm{~K}$. In DPSM, the number of the point sinks for one droplet is chosen as $M=200$.

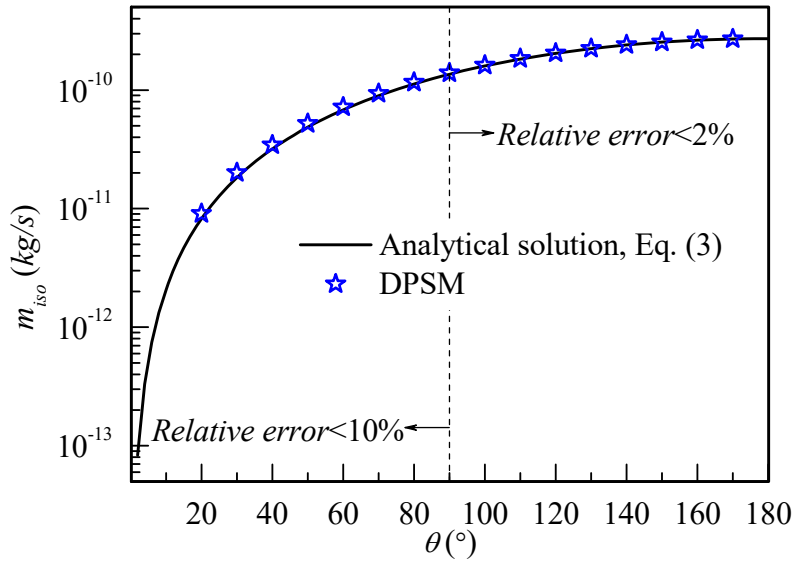

Fig. 3. The predicted condensation rate by DPSM and the analytical solution for an isolated growth droplet.
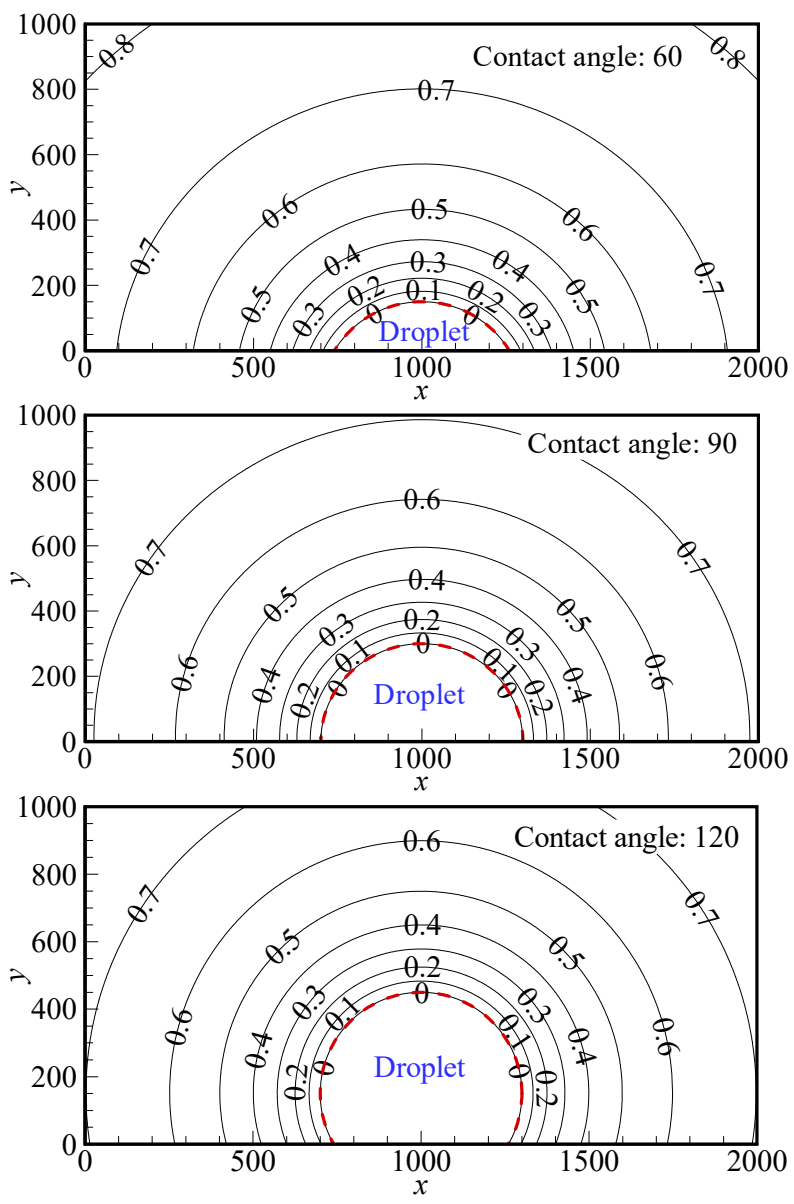

Fig. 4. The normalized vapor concentration fields for single condensing droplet in different contact angles (unit: $\mu \mathrm{m}$ ).

Fig. 3 plots the predicted condensation rate comparing with the analytical solutions by Eq. (4). It can be observed that the predictions by DPSM closely match the analytical solution. The relative error between two manners, for the contact angle greater than $90^{\circ}$, remains below $2 \%$. And the relative error falls within $10 \%$ for the smaller contact angle. Furthermore, the dimensionless concentration distributions of the exterior domain surrounding the droplet are provided by DPSM. For selected three contact angles $\left(60^{\circ}, 90^{\circ}, 120^{\circ}\right)$, the dimensionless concentration fields are presented in Fig. 4 from the cross-sectional view. The red dashed circular line depicts the droplet profiles. Based on the uniqueness theorem, an evaluation criteria whether or not certain boundary conditions is satisfied is utilized to evaluation the accuracy. It is apparent through the zero isoconcentration lines overlapping with the droplet profiles that the desired boundary conditions are satisfied exactly in different contact angles.

\subsection{A binary array of droplets}

This section aims to explore the sensitive extent of the droplets size and inter-spacing for the interaction effects of droplets. For that, the DPSM method is utilized to predict the condensation behavior of a pair of neighboring droplets. Two droplets located in the normalized concentration field have the contact angle 90 deg, the radius $R_{1}$ and $R_{2}$ and they are separated by a distance $L$. Their condensation rates $m_{s y s}$ are predicted by the DPSM method with $M=200$. Based on the growth of isolated form, the condensation rate at the corresponding size $m_{\text {iso }}$ is calculated by Eq. (4).

The relative size of droplets affecting the interaction effect is investigated by keeping a constant size of droplet $2 R_{2}=100 \mu \mathrm{m}$ and a decreasing size of droplet 1 $R_{I}$ from $100 \mu \mathrm{m}$ to $10 \mu \mathrm{m}$. And the effects of the interdroplet space are further considered by changing the droplet location. Figure 5 plots the predicted correction factor against the inter-droplet space for all of the cases considered. And the normalized concentration fields for different cases are further presented in Fig. 6 and Fig. 7 aiming to examine the interaction effect in affecting the condensation behavior.

Actually, the correction factor defined by Eq. (3) reflects the intensity of the inter-droplet interaction which can directly affect the condensation rate. A smaller correction factor means the strong interaction which significantly reduces the droplet condensation rate; otherwise the weak interaction effect can be dismissed $(\eta \rightarrow 1)$. All cases presented in Fig. 6 show that the interaction effects are strongly sensitive for the relative size of the droplets. The condensation rate of the smaller droplet is significantly reduced due to a strong interaction as its size decreases gradually. Comparatively, the reduction of the condensation rate due to the interaction effect is getting smaller for the larger droplet. For example, at $R_{2}=10$ and $R_{1}=100 \mu \mathrm{m}$, the correction factor for the small droplet decreases up to $\eta_{1}=0.2$, while the large droplet has a correction factor tending to unit one for all cases at this relative droplet size. In addition, the inter-droplet distance also plays a significant role in affecting the condensation rate. It can also be observed that the correction factor $\eta$ is an inverse function of the 
separation distance $L$. With increasing $L$, the correction factor tends to $\eta=1$, which means that the droplet has a negligible interaction with each other. When the droplets become closer, the strong interaction with each other urges the correction factor to decrease.
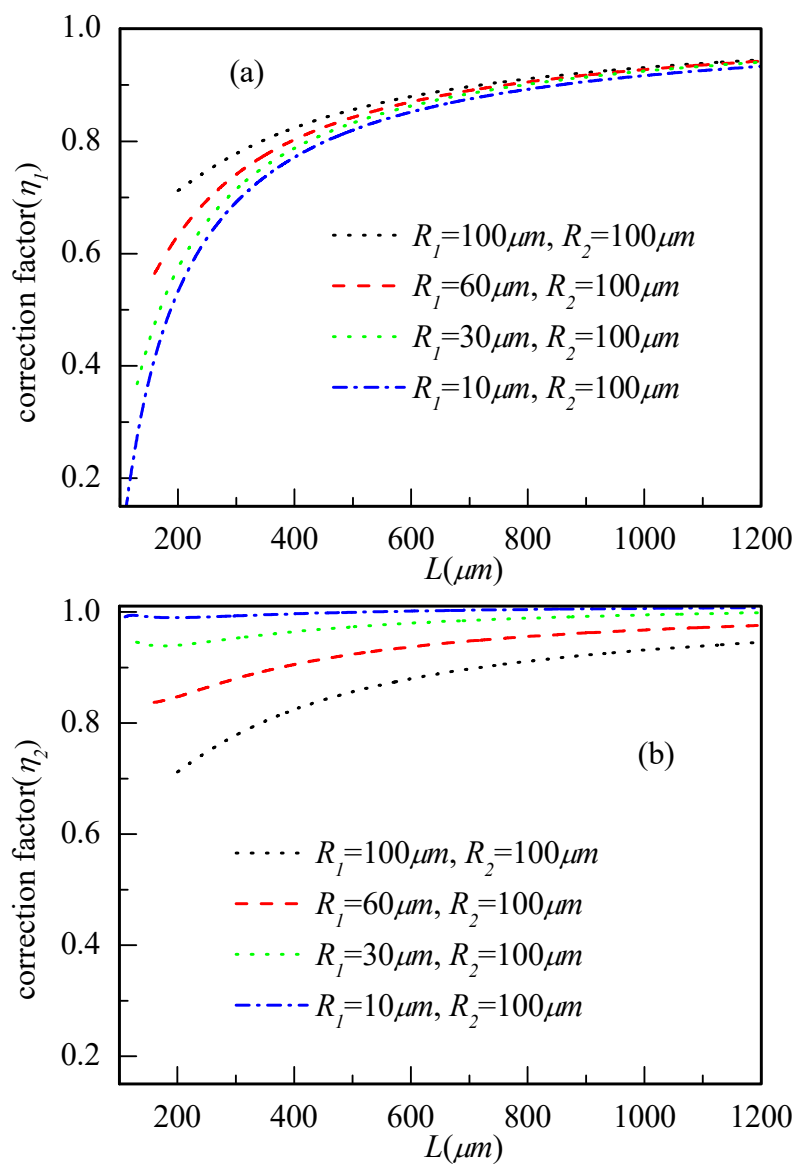

Fig. 5. The correction factors related to the condensation rate. (a) droplet No. 1, $R_{1}=10-100 \mu m$; (b) droplet No. 2, $R_{2}=100 \mu \mathrm{m}$.

Those issues shown by the correction factor can be further explained by Fig. 6 and Fig. 7. It can be found that the neighboring droplet leads to the local depletion of vapor in the direction toward each other; hence, the droplet condensation rate is reduced due to the decreased concentration gradient normal to the droplet surface. When two droplets have the same size, the symmetric region of the vapor depletion in Fig. 6 can explain the same curve of the correction factor for two droplets shown in Fig. 5. For different sizes of droplets, the dimensionless concentration distributions by Fig. 7 indicate that the smaller droplet is almost entirely located in the zone of the vapor depletion. In comparison, the surface of the larger droplet partly faces to the depletion zone. Therefore the condensation behavior of the smaller droplet is easily affected by the local depletion of vapor. As two droplets closing, the vapor depletion becomes severe gradually.

To sum it up, the strength of the inter-droplet interaction which attributes the reduction of the droplet condensation rate is sensitive to the relative size of droplets and their inter-distance. The local depletion of vapor because of the coming neighbors explains the reduction of condensation rate. Those revealed phenomena in this work keep consistent with that reported in the literatures as presented in the first section.
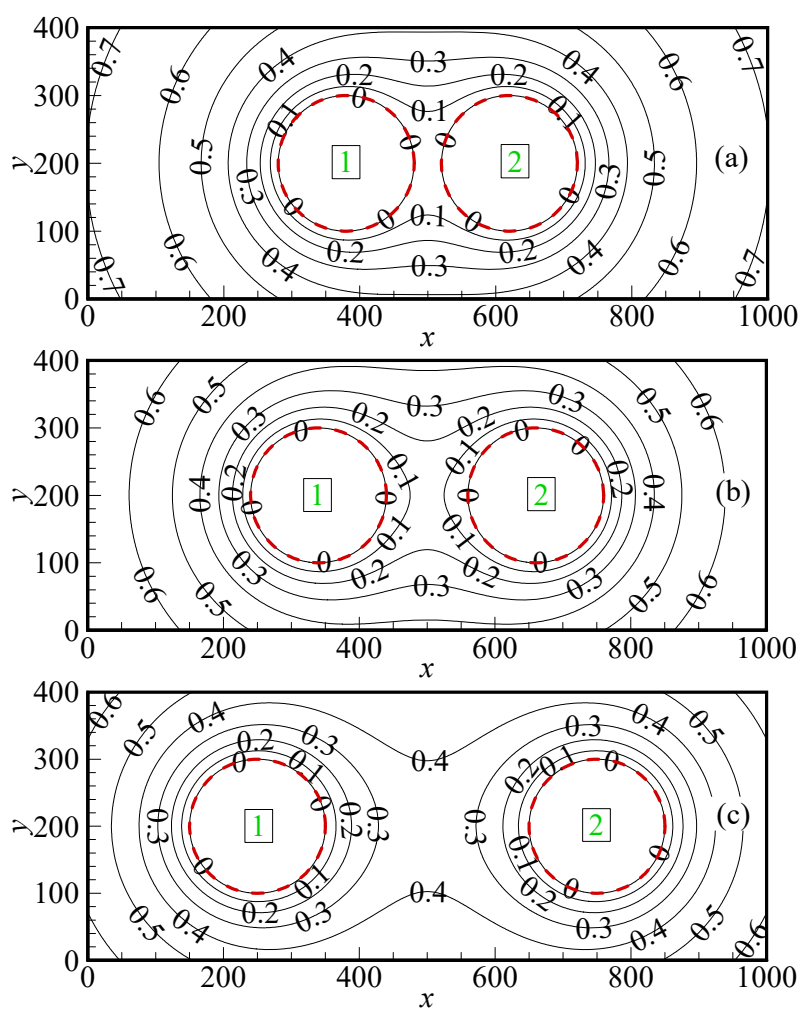

Fig. 6. The normalized vapor concentration distributions using the DPSM method for two condensing droplets $R_{1}=R_{2}=100$ $\mu m$. (a) $L=240 \mu \mathrm{m}$; (b) $L=320 \mu \mathrm{m}$; (c) $L=500 \mu \mathrm{m}$.
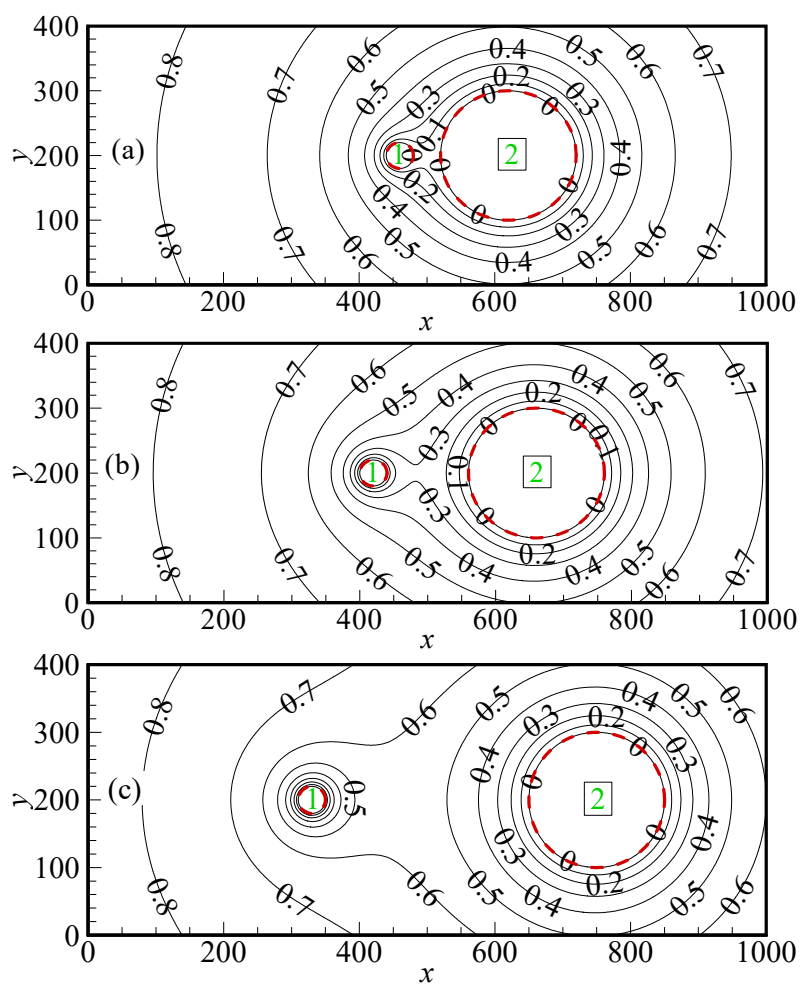

Fig. 7. The normalized vapor concentration distributions using the DPSM method for two condensing droplets having different sizes $R_{1}=10 \mu \mathrm{m}$ and $R_{2}=100 \mu \mathrm{m}$. (a) $L=160 \mu \mathrm{m}$; (b) $L=240 \mu m$; (c) $L=420 \mu m$. 


\subsection{A characteristic droplet array during dropwise condensation}

During dropwise condensation, a large number of closely neighboring droplets distributed over the substrate and spreading in a wide size range bring strong interactions, which will apparently reduce the condensation rate. Therefore, the interaction effects between droplets play an important role in affecting the overall condensation behaviors.

In order to examine the role of the inter-droplet interaction in dropwise condensation, a characteristic spatial and size distribution of droplets from dropwise condensation experiment is further considered, as the snapshot $(9400 \times 6800 \mu \mathrm{m})$ recorded by the microscope camera and shown in Fig. 8. After the image-post processing, 1167 droplets having the radius range 20$1090 \mu \mathrm{m}$ are detected and plotted by the red circle. All droplets are assumed as a spherical cap with the contact angle $90^{\circ}$.

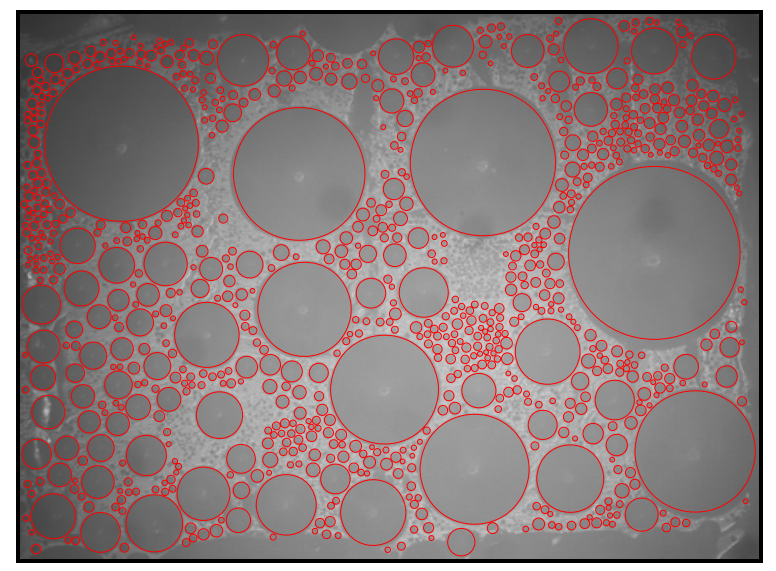

Fig. 8. A characteristic droplet array during dropwise condensation.

Considering the droplet distribution in Figure 8, the DPSM method is utilized to predict the interaction effects between droplets. In DPSM, the number of the point sinks for each droplet is chosen as $M=200$ in light of the computation cost and the solution accuracy. And the GPBiCG and TwoD algorithms are respectively considered for the solution of the linear system in Eq. (8) and the evaluation of the surface integral in Eq. (12). The numerical errors for the GPBiCG and TwoD algorithms are set as 1e-5 and 1e-6, respectively. The predicted correction factors of droplets are plotted in Fig. 9(a) as a function of its radius. Furthermore, including the size and position information of droplets, Fig. 9(b) plots the droplets filled by the color corresponding its correction factor.

From Fig. 9(a), the correction factors denote that the droplet condensation rate is reduced 3-1000 times due to the interaction effect. In comparison, the small size droplets are easily affected by the neighboring large droplets. Thus, smaller droplet totally has a smaller correction factor due to the strong interaction. However, the reduction of the condensation rate because of the interaction effect is not absolutely driven by its size. As shown in Fig. 9(b), having an approximately equal size, three droplets marked by the numbers present different interaction intensity. As a result, the droplets locating on the substrate center have a stronger interaction than the droplets closing toward the substrate edge, which has been observed during experiments [7]. Actually, the interaction effects of droplets, in affecting the behavior of dropwise condensation, is a complicated issue and mainly depended on the spatial and size distributions of droplets. In conclusion, the inter-droplet interactions present personality as an independent individual, and have further generality on the whole.
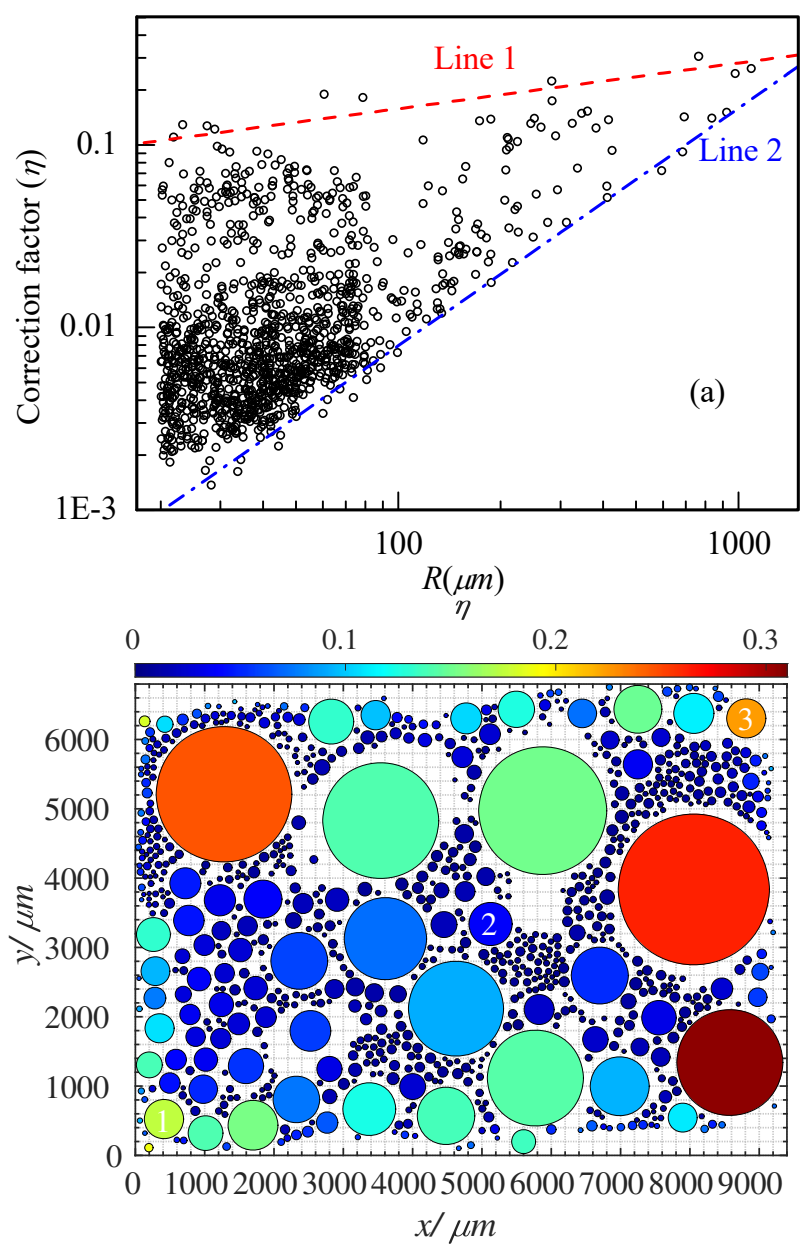

Fig. 9. (a) the predicted correction factors of the droplets by DPSM versus its size; (b) the droplets filled by the color according to their correction factor.

\section{Conclusions}

In order to evaluate the inter-droplet interaction in affecting the behavior of dropwise condensation, a distributed point sink method is developed without requiring solution of the diffusion equation and the numerical discretization. As the method of Green's function, the boundary condition can be satisfied automatically due to its nature.

In handling the growth of an isolated droplet, the predicted condensation rates by the DPSM method show a good agreement with the analytical solutions at different contact angles. In case of the representative droplets array, the investigations indicate that the interaction effects of droplets as a complicated issue are entirely depended on the spatial and size distributions of 
droplets. Owing to the local depletion of the vapor, the interaction effects between droplets play a significant role in reducing the droplet condensation rate.

The authors want to thank the financial support from China Scholarship Council (CSC) and German Research Foundation (DFG, Grant No. Gr1060/18).

\section{References}

1. J. Huang, J. X. Zhang, L. Wang, Appl. Therm. Eng., 89, 469-484 (2015).

2. X. H. Ma, X. D. Zhou, Z. Lan, Y. M. Li, Y. Zhang, Int. J. Heat Mass Transfer 51, 1728-1737 (2008).

3. S. Khandekar, K. Muralidhar, Dropwise condensation on inclined textured surfaces (Springer, New York, 2014).

4. J. W. Rose, Proc. Inst. Mech. Eng., Part A: J. Power Energy 216, 115-128 (2002).

5. S. Zheng, F. Eimann, C. Philipp, T. Fieback, U. Gross, Int. J. Heat Mass Transfer 120, 879-894 (2018).

6. S. Zheng, F. Eimann, C. Philipp, U. Gross, Proc. 16th Heat Transfer Conf., 2133-2140 (2018).

7. M. G. Medici, A. Mongruel, L. Royon, D. Beysens, Phys. Rev. E 90, 062403 (2014).

8. I. O. Ucar, H. Y. Erbil, Colloids Surf. A: Physicochem. Eng. Asp. 411, 60-68 (2012).

9. R. N. Leach, F. Stevens, S. C. Langford, J. T. Dickinson, Langmuir 22, 8864-8872 (2006).

10. J. E. Castillo, J. A. Weibel, Int. J. Heat Mass Transfer 118, 708-719 (2018).

11. J. E. Castillo, J. A. Weibel, Int. J. Heat Mass Transfer 133, 641-651 (2019).

12. S. Zheng, F. Eimann, C. Philipp, T. Fieback, U. Gross, Int. J. Heat Mass Transfer 141, 34-47 (2019).

13. K. Annamalai, W. Ryan, Prog. Energy Combust. Sci. 18, 221-295 (1992).

14. M. Labowsky, Chem. Eng. Science 31, 803-813 (1976).

15. D. Placko, T. Kundu, DPSM for modeling engineering problems (John Wiley \& Sons Inc., New Jersey, 2007).

16. S. Fujino, Appl. Numer. Math. 41, 107-117 (2002).

17. L. F. Shampine, Appl. Math. Comput. 202, 266-274 (2008). 